\title{
Couvrir une actualité de crise terroriste : un dispositif « web first »?
}

Les cas du «Brussels Lockdown » et des attentats de Bruxelles

French-speaking belgian news websites coverage during the "brusselslockdown" and brussels terror attacks: a "web first" set?

Cobertura del "brussels lockdown" y de los atentados de bruselas por los sitios web de actualidad belgas francófonos: ¿un dispositivo "web first"?

\section{Quentin Jardon, Sandrine Puissant Baeyens et Lara Van Dievoet}

\section{(2) OpenEdition}

\section{Journals}

Édition électronique

URL : http://journals.openedition.org/ctd/722

DOI : $10.4000 /$ ctd. 722

ISSN : 2491-1437

Éditeur

Chaire Unesco Pratiques émergentes en technologies et communication pour le développement

Référence électronique

Quentin Jardon, Sandrine Puissant Baeyens et Lara Van Dievoet, "Couvrir une actualité de crise terroriste : un dispositif « web first »? », Communication, technologies et développement [En ligne], 4| 2017, mis en ligne le 04 septembre 2017, consulté le 02 avril 2021. URL : http:// journals.openedition.org/ctd/722; DOI : https://doi.org/10.4000/ctd.722

Ce document a été généré automatiquement le 2 avril 2021.

Communication, technologies et développement 


\section{Couvrir une actualité de crise terroriste : un dispositif « web first »?}

Les cas du « Brussels Lockdown » et des attentats de Bruxelles

French-speaking belgian news websites coverage during the "brusselslockdown" and brussels terror attacks: a "web first" set?

Cobertura del "brussels lockdown" y de los atentados de bruselas por los sitios web de actualidad belgas francófonos: ¿un dispositivo "web first"?

Quentin Jardon, Sandrine Puissant Baeyens et Lara Van Dievoet

1 La nuit du 20 novembre 2015, une semaine après les attentats qui ont frappé Paris le 13 novembre, l'Organe de Coordination pour l'Analyse de la Menace (OCAM), en Belgique, évalue la menace terroriste comme « sérieuse et imminente » dans la capitale et place la Région bruxelloise en niveau d'alerte terroriste maximal : quatre sur une échelle de quatre. Alors que la présence policière et militaire y est renforcée, les grands événements sont annulés et les écoles et stations de métro fermées. De nombreux commerces restent également portes closes. Le 26 novembre, le niveau de menace descend à trois, marquant la fin de la période baptisée Brussels Lockdown, ou fermeture de Bruxelles. Le 22 mars 2016, la capitale belge, toujours en état d'alerte de niveau trois, connaît des explosions à l'aéroport de Zaventem et à la station de métro Maelbeek qui font 32 morts et 340 blessés.

2 Ces deux événements ont donné lieu à une couverture médiatique intense de la part des médias belges. Éditions spéciales et lives se multiplient sur leurs sites internet pour couvrir l'actualité en continu. Le nombre de visites sur tous les sites d'information francophones belges augmente de manière significative. Les données certifiées par le Centre d'Information sur les Médias (CIM) ${ }^{1}$ montrent que, le 13 novembre 2015, un premier pic apparait avec les attentats de Paris, suivi d'un second la semaine suivante lors du Lockdown de Bruxelles. Entre les deux, les chiffres restent sensiblement plus 
élevés que la moyenne. Sans surprise, des audiences encore plus importantes ont été mesurées lors des attentats du 22 mars 2016.

3 En plus de croître, l'audience web se restructure lors de ces deux crises. Si tous les médias connaissent un pic, les sites des médias nationaux dits "de référence» enregistrent une hausse plus importante que celle de leur concurrence ${ }^{2}$ Le rapport entre le nombre de visites du 21 et celles du 22 mars montre que leur public quadruple ou quintuple en un jour, tandis que le rapport se situe entre deux et trois pour les sites d'information régionale du groupe Sudpresse et de L'Avenir, et autour de 1,5 pour les sites liés au quotidien économique L'Écho et à l'hebdomadaire Le Vif/L'Express, aux publics plus ciblés. Les sites régionaux de Sudpresse sont même dépassés par leurs concurrents, alors qu'ils sont habituellement les plus visités. Seuls Bxi.be et DH.be semblent se démarquer de la tendance des médias régionaux ${ }^{3}$ Ces disparités et le pouvoir d'attraction plus important des titres « de référence " peuvent être interprétés comme le signe d'une demande accrue de fiabilité dans un contexte de crise, saturé d'informations. Mais ces différences relèvent également, en partie, de la plus forte capacité des médias «nationaux de référence » à attirer un lectorat étranger ${ }^{4}$

4 La littérature suggère que si les routines professionnelles sont centrales dans le dispositif de crise des journalistes, elles ne suffisent pas seules pour réagir de façon appropriée face au caractère exceptionnel de ce type d'événements (Berkowitz, 1992 ; Konow Lund et al., 2016). Ce constat, couplé à l'importance de l'augmentation et de la reconfiguration des audiences web, nous semble justifier l'observation des rédactions en ligne dans un contexte de crise. Notre étude se penche sur la place accordée au Web dans l'organisation des rédactions des médias francophones d'information générale belges dits "traditionnels ${ }^{5}$ " durant la couverture du Lockdown et les attentats de Bruxelles et sur le rôle des équipes web dans la production de contenus. Comment ces rédactions bimédias ou plurimédias se sont-elles organisées? Quel mode d'opération ont-elles privilégié ? Ont-elles donné la priorité au Web ou se sont-elles concentrées sur leur activité historique ? Quel type de contenu a-t-il été produit pour le Web?

Figure 1. Évolution du nombre de visites des sites d'informations belges francophones (14-31 mars 2016) 


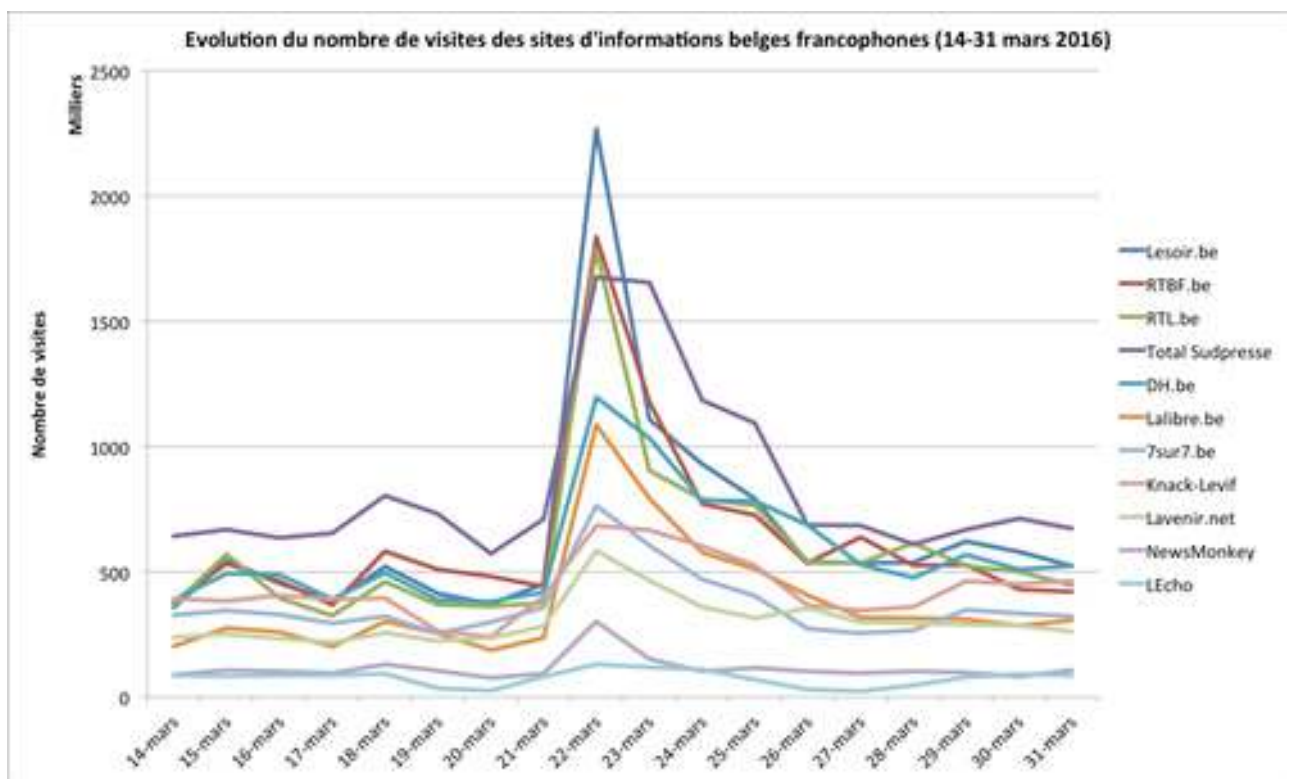

Le pic d'audience enregistré le 22 mars 2016 est plus important pour les médias d'information générale dits « de référence » et « nationaux » : Lesoir.be, RTBF.be, RTL.be et LaLibre.be.

Source : CIM

\section{Neuf rédactions}

Afin de répondre à ces questions, nous avons mené deux vagues de neuf entretiens semi- directifs avec les responsables éditoriaux des sites internet de l'ensemble des principaux acteurs "traditionnels» du marché de l'information générale belge francophone. Nous avons rencontré à deux reprises les rédacteurs en chef des deux sites web des quotidiens dits "nationaux" et "de référence", Le Soir et La Libre Belgique, ceux des sites des deux principaux groupes de quotidiens régionaux L'Avenir et Sudpresse ${ }^{6}$, celui des supports numériques du quotidien économique L'Écho, celui de l'hebdomadaire d'information générale Le Vif/L'Express, celui du site d'information de la radio télé de service public RTBF et celui de son concurrent direct, le site du média audiovisuel privé RTL Belgique. Afin d'intégrer également une télévision locale dans notre étude, nous y avons ajouté la rédaction web de la télévision bruxelloise $B \times 1^{7}$

Le marché francophone belge ne compte qu'un seul pure player ${ }^{8}$, Newsmonkey, exclu de cette étude afin de maintenir la cohérence de notre corpus. Toutes les rédactions web observées sont donc adossées à un support « traditionnel » papier, radio ou télévision.

La première vague d'entretiens, réalisée en janvier et février 2016, portait sur la couverture du Lockdown par les sites internet des médias sélectionnés. Suite aux attentats de Bruxelles, nous avons réalisé en mai et juin 2016 une deuxième vague d'entretiens au sein des mêmes rédactions. Afin d'étudier la couverture de ces deux crises d'un point de vue organisationnel, le recours à des entretiens semi-directifs nous a semblé approprié. Ce type d'entretien permet «l'analyse du sens que les acteurs donnent à leurs pratiques et aux événements auxquels ils sont confrontés: leurs systèmes de valeurs, leurs repères normatifs, leurs interprétations de situations conflictuelles ou non, leurs lectures de leurs propres expériences, etc.» (Quivy et al., p. 196, 995). Si cette méthode ne comprend pas l'observation directe de l'action des responsables éditoriaux en période de crise, elle constitue le meilleur moyen disponible a posteriori pour approcher leur 
lecture de ces événements et les moyens mis en œuvre. Les entretiens constituent, en outre, une source d'information riche sur les réflexions et intentions des responsables web lors des deux crises étudiées.

\section{Organisation des équipes rédactionnelles : composer dans l'urgence}

8 La première partie de notre analyse concerne l'organisation des rédactions durant le Brussels Lockdown et les attentats de Bruxelles. Comment les rédactions web se sont-elles structurées? Quelle est la place des équipes web dans les médias "traditionnels » durant ce type d'événements? Disposent-elles de feuilles de conduite pour la couverture d'attentats terroristes? L'ensemble des données récoltées nous a permis de mettre en évidence que si la succession de crises permet l'acquisition de réflexes, les dispositifs mis en place le sont de manière spontanée et non formalisée.

De manière générale, le Web ne semble pas disposer d'un nombre plus important de règles spécifiques aux situations d'urgence que le reste de la rédaction. Plusieurs responsables mentionnent cependant une vigilance accrue, en particulier envers les contenus provenant des réseaux sociaux. Ils insistent sur la nécessité de prendre le temps nécessaire à la vérification d'une information, malgré l'urgence.

Lors de ces deux événements, les rédactions web ne restructurent pas en profondeur leur fonctionnement, mais l'adaptent aux contraintes. Elles mobilisent pigistes et journalistes spécialistes d'un sujet ou travaillent plus longtemps, par exemple. Un renforcement qui semble spontané, puisque la majorité des responsables éditoriaux expliquent qu'ils n'ont pas dû rappeler leurs équipes pour que celles-ci se mobilisent. En plus de ce renforcement des effectifs, les rédactions web tentent d'augmenter leur efficacité en affectant l'ensemble de leurs journalistes à la couverture de l'événement.

11 La réorganisation de la rédaction se base sur le «bon sens" et "l'autonomie » des journalistes. Seul un responsable, sur les neuf interrogés, déclare disposer d'un document interne établissant une procédure d'urgence en cas d'actualité exceptionnelle de ce type. Quatre rédactions affirment n'avoir jamais effectué de débriefing de leur couverture du Lockdown ou des attentats. La plupart de celles qui l'ont fait n'ont pas rendu officiels leurs apprentissages : seule une rédaction web a déclaré avoir rédigé une feuille de route pour valider les contenus issus des réseaux sociaux et formaliser les sources nécessaires à la veille d'information en période de tension. La structuration du dispositif d'urgence correspond davantage à l'acquisition de réflexes au fil des crises successives, par itération et sans formalisation particulière. "On ne note pas nos expériences. C'est de la mémoire vive. Si la moitié de l'équipe se barre, c'est perdu... » (Vif.be)

12 La coordination des responsables éditoriaux joue un rôle important dans ces momentsclés. Bx1 a connu un changement de rédacteur en chef entre le Lockdown et les attentats, ce qui a "réorganisé le pilotage » de crise. S'ils ne décrivent pas un dispositif d'urgence conscient et intentionnel, les médias rebondissent sur des stratégies et des automatismes mis en place dans le cadre de l'actualité «habituelle» ou issus de la répétition de crises. Deux responsables web ont souligné que cette absence de règles leur permettait, selon eux, d'être plus réactifs et efficaces. 

restent au cœur du fonctionnement des rédactions en période de crise. Plusieurs études ont, en effet, mis en évidence le fait que les tâches journalistiques exceptionnelles ne mènent pas à de nouvelles formes d'organisation de la rédaction, mais que l'improvisation se construit sur l'adaptation des routines existantes (Berkowitz, 1992; Konow Lund et al., 2016) Ces études soulignent aussi le rôle central des expériences précédentes d'événements similaires. (Olsson, 2009) Koljonen et Väliverronen (2011) se servent du concept de reflective professional practice, ou pratique professionnelle réflective, selon laquelle les professionnels apprennent à partir de leur expérience. D'après les journalistes qu'ils ont interrogés, il est « presque impossible de s'interroger sur ses propres actions et leurs bases en plein travail mouvementé sans compromettre leur travail» (Koljonen et al., 2011, p. 721). Olsson (2009) souligne en outre, elle aussi, l'importance du management, de sa perception et de son système de valeur dans la réaction.

\section{La hiérarchie des supports}

14 Il est important de rappeler que les sites d'information étudiés ne sont pas des pure players et ne sont donc pas complètement indépendants de rédactions papier ou audiovisuelles. L'analyse des entretiens suggère la prévalence d'un dispositif web first durant ces deux événements: de manière générale, l'intention est de publier les informations immédiatement sur le Web pour informer en continu (Grabowicz, 2014). Dans son étude du journalisme web en Belgique, Amandine Degand observe que cette forme d'organisation s'inscrit déjà dans les habitudes des rédactions. "Puisque le journalisme en ligne se développe et mise, bon gré mal gré, sur limmédiateté, les médias traditionnels doivent à leur tour se repositionner, pour ne pas faire la même chose que leurs collègues du Web. (...) C'est la politique du "Web first" telle que pratiquée par la plupart des médias belges aujourd'hui. L'information doit d'abord paraître sur le net, avant d'être commentée, enrichie et complétée sur un autre support ». (Degand, 2012, p. 305)

Pendant le Lockdown et les attentats de Bruxelles, les échanges et les synergies entre supports traditionnels et online ont été jugés plus importants qu'en temps normal par la majorité des rédacteurs-en-chef interrogés. Cependant, lors du Lockdown et des attentats, les renforts humains ne se dirigent pas prioritairement sur le Web, mais semblent se répartir sur l'ensemble de la rédaction. Dans la majorité des cas, il semble même qu'ils se dirigent en priorité vers les supports papier, radio ou télévisé, malgré l'exigence d'instantanéité plus importante du Web. «Le bimédia [synergies avec le Web] fonctionne très bien dans le cadre d'une actualité "normale", mais en cas de coup de feu, les journalistes radio sont à $110 \%$ pour la radio et les journalistes télé sont à $110 \%$ pour la télé. Donc qu'est-ce qu'on fait sur le Web?» (RTBF.be) Les journalistes web peuvent même être amenés à retraiter l'information diffusée par les médias traditionnels : à la RTBF et chez RTL, pendant les attentats, un journaliste web se consacre entièrement à retranscrire ce qu'il entend sur antenne. S'il y a un journal ou magazine à produire, cela restera la priorité: «Le Web produit plus vite et plus, mais sans empiéter sur les moyens mis au journal. " (Lecho.be) À RTL par exemple, le journaliste en charge de la veille de l'actualité travaille habituellement pour tous les supports, mais bascule en télévision pour alimenter le direct au moment des attentats. Au Vif/L'Express, les renforts vers le Web

Communication, technologies et développement, 4 | 2017 
ont été plus nombreux pendant le Lockdown que lors des attentats, parce que les journalistes disposaient de plus de temps avant l'échéance de bouclage du magazine.

Ces processus ne sont pas figés. Les rédactions tentent de s'adapter d'un événement à l'autre. Le responsable du Soir.be explique, par exemple, que sa rédaction a tiré les leçons d'une crise précédente, durant laquelle l'équipe web avait été réduite à une seule personne, livrée à elle-même, pendant que les autres journalistes étaient en conférence de rédaction: "il n'y a plus jamais de soucis de manque d'effectifs, bras, ressources, pour alimenter le Web le plus correctement possible." Nous pouvons parler à nouveau, ici, de reflective professionnal practice (Koljonen \& al., 2011).

\section{Spécificité des contenus web}

La suite de notre analyse concerne les contenus web produits par les rédactions dans un contexte de couverture continue et en urgence. Les entretiens menés dans les neuf rédactions étudiées révèlent deux types de contenus pour alimenter les sites d'information: le contenu produit par les journalistes et le contenu dit "citoyen", produit par des non-journalistes. Nous nous sommes intéressés à la nature de ces deux types de contenus dans le traitement de l'actualité lors du Brussels Lockdown et des attentats de Bruxelles.

\section{Contenu « professionnel »}

Dans tous les cas, les médias mettent en place un live $e^{9}$, ou fil d'actualité en continu, alimenté par la rédaction dès qu'elle obtient une nouvelle information. Ce live peut parfois être ouvert sur une longue période, sans interruption (trois jours, par exemple, sur Levif.be), permettant de donner la dernière information sans devoir écrire un nouvel article. À L'Écho, il est enrichi des premières analyses pour se détacher de la dépêche sèche : l'instantanéité, "c'est un compromis entre la rapidité et la vérification". Utilisé également hors période de crise, mais particulièrement adapté à la situation de breaking news en développement, ce format permet au public de vivre les événements en temps réel.

Au-delà de l'instantanéité, les différentes rédactions insistent sur ce qui fait l'essence de l'information en ligne, particulièrement en cas d'événement fort : le témoignage et les images. Le témoignage, qu'il soit sous forme de texte ou d'image, vient incarner les informations livrées souvent via un compte-rendu factuel, notamment à travers les live. Le Web, lors d'une alerte d'actualité, c'est "le règne de l'image " (RTLinfo.be). Il doit être lisible, accessible et utile (LEcho. be). À cette fin, certaines rédactions ont mis en place un digest, soit un encadré rappelant les faits essentiels, mis en bonne place au sein de tous les articles qui traitent de l'événement concerné. Ce digest est également une manière de s'adapter au comportement de navigation des internautes, qui pour une majorité n'accèdent pas à un article via la homepage du site, mais via une plateforme externe comme Facebook ou Google.

Le web first, déjà présent hors période de crise, s'impose dans les rédactions papier. Nous constatons, dans les propos des représentants de rédactions web adossées à des médias de presse écrite, le renforcement de la distinction entre l'information produite pour le Web et celle rédigée pour la version papier. Lors du Lockdown comme suite aux attentats de Bruxelles, le Web est privilégié pour le direct, l'image et le témoignage, 
quand le papier propose des analyses et des reportages plus longs. Chez Sudpresse, le site se nourrit de photos, de vidéos et de faits. C'est aussi le cas pour le papier, mais les journalistes tentent d'y prendre plus de recul. Les papiers longs n'ont pas leur place sur le site de Sudpresse. À L'Écho, on valorise la rapidité de l'info sur le Web, tandis que le papier cherche un angle spécifique et y consacre le temps nécessaire, si l'actualité le permet. Les autres titres de presse écrite rencontrés ont adopté la même logique. L'Avenir se démarque, affirmant publier parfois des articles plus longs sur le site que dans l'édition papier, mais reconnaît que ce cas de figure se présente plus rarement lors d'événements graves et imprévus. Notons que L'Écho s'est démarqué de ses concurrents lors du Brussels Lockdown, en publiant une édition exclusivement numérique, à destination des tablettes et mobiles, le soir du 22 novembre.

Du côté des médias audiovisuels, le rapport entre le contenu web et le contenu télé/ radio est moins clair. Les sites récupèrent le flux diffusé sur antenne (RTLinfo.be et RTBF.be), auquel est ajouté ce qui ne méritait pas sa place dans le canal de diffusion traditionnel (Bx1.be) : témoignages, vidéos amateurs, etc. À tel point qu'à l'heure du journal télévisé suivant, le public sait déjà de quoi on va parler (RTLinfo.be). Les médias traditionnels ont dès lors tout intérêt à travailler sur la mise en perspective, le reportage et les analyses d'expert, en déployant un format de plus en plus proche du magazine. Cette pratique semble se renforcer (RTLinfo.be). Certains médias audiovisuels, comme Bx1, ont par contre clairement opté pour une stratégie web first: "Dès qu'on avait une information, elle partait sur le Web, avant d'être à l'antenne. Parce que ça va plus vite. C'est la raison du Web. La souplesse de l'outil permet de diffuser l'info le plus vite possible. Il n'y a pas de raison d'attendre que ce soit passé au journal. » (Bx1.be)

Si l'on peut s'attendre à une utilisation importante des smartphones en période de crise, leur usage par les journalistes s'est néanmoins révélé limité ou empêché lors du Lockdown et des attentats de Bruxelles, faute d'avoir quelque chose à montrer. En novembre 2016, les rues sont vides. Lors des attentats du 22 mars, l'installation d'un périmètre de sécurité autour de l'aéroport de Zaventem et de la station de métro Maelbeek a refoulé journalistes et badauds à distance des lieux de la tragédie, ne leur permettant pas de filmer ou photographier grand-chose, si ce n'est un ballet d'ambulances et de militaires armés. Les seules photos et vidéos disponibles sont celles prises par des témoins durant les quelques minutes, voire dizaines de minutes, qui ont suivi les différentes explosions.

\section{Contenu « citoyen »}

23 Nous nous attendions à ce que les rédactions web recourent aux contenus produits par des non professionnels pour pallier leur absence sur les lieux au moment des attentats (Bruno, 2010). Les rédactions ont bien écumé les réseaux sociaux à la recherche d'une photo ou d'une vidéo digne de publication, mais elles affirment pourtant n'avoir publié qu'un nombre limité de contenus amateurs, malgré la gravité des événements et leur aspect spectaculaire.

La sélection de ce type de contenus et la notion de plus-value par rapport à ce que les journalistes pourraient fournir par eux-mêmes varient d'une rédaction à l'autre. Les responsables saluent la possibilité d'être "à plein d'endroits à la fois» (RTLinfo.be), de donner à voir une situation où le média n'était pas présent. Ces images amateurs permettent de transmettre une émotion là où les mots ne suffiraient pas (LEcho.be), 
elles sont intéressantes, car frappantes (RTLinfo.be), comme la vidéo tournée juste après les explosions dans l'aéroport de Zaventem ${ }^{10}$, où l'on peut ressentir "l'atmosphère surréaliste " sans passer par des images sanguinolentes. Mais elles ne méritent une diffusion que si elles portent une "réelle info» (Levif.be). Les déclarations des responsables web rejoignent la conclusion de Nicolas Bruno dans son étude sur l'impact de l'information en temps réel sur la couverture des crises: l'importance de la vérification et la nécessité d'offrir un contenu fiable et pertinent (Bruno, 2010-2011) ${ }^{11}$ Certains responsables des sites d'information insistent sur le caractère délicat des photos et vidéos d'amateur en contexte d'urgence: les pièces visuelles sont parfois difficiles à vérifier, et une erreur peut être très dommageable pour le média. Plusieurs rédactions se sont d'ailleurs fait piéger dans la matinée du 22 mars par la vidéo d'une explosion à l'aéroport de Moscou plusieurs années auparavant, l'identifiant comme celle de Zaventem. La procédure de vérification de l'information de contenu citoyen est encore intuitive. Si Bx1 était en train de mener une étude juridique pour établir une ligne de conduite générale, la plupart des médias prennent la décision de diffuser ou de ne pas diffuser au cas par cas, en passant le fichier au crible, sans disposer de charte ou de règles.

\section{Le paradoxe du « web first »}

Lors du Brussels Lockdown et des attentats du 22 mars, on assiste à une convergence des moyens et des contenus vers un seul sujet et ses déclinaisons : le « crisis news event » ou actualité de crise, un événement surprise qui remet en question les valeurs organisationnelles et exige une réponse immédiate (Olsson, 2010). Le Web est particulièrement adapté à ce type d'événements. Il permet à la fois de gérer la surprise et la nécessité de réponse immédiate, car il rend possible l'enrichissement progressif de contenus en cours de développement.

Le Web permet de faire du direct au moment où le public exprime - d'après les chiffres d'audience exposés supra - le besoin permanent d'être informé. Les rédactions tentent donc de répondre à cette demande d'instantanéité et de fiabilité en appliquant une politique web first. D'une façon générale, on observe que publier des informations rapidement en ligne devient une priorité lors du Lockdown et des attentats de Bruxelles. En plus d'articles et comptes-rendus, le live, les images et les témoignages ont pris une place prépondérante sur les sites web des médias traditionnels en Belgique francophone. Un nombre limité de contenus rapportés par des citoyens à l'aide de leur smartphone sont également publiés.

27 Cependant, peu de moyens humains supplémentaires peuvent être dégagés pour le Web dans les rédactions «traditionnelles ». La notion de web first doit donc être nuancée : il s'agit plus d'une complémentarité entre supports, dans une logique bi- ou plurimédia que d'une réelle prévalence du Web. La nécessité d'informer immédiatement prime, peu importe le support. Si le papier, publié avec un délai, n'a d'autre choix que de recourir au Web pour informer son public en temps réel, les éditions spéciales radio et télévisées en continu ont pu côtoyer et parfois nourrir les lives des sites web.

On constate que la convergence autour du crisis news event bouscule les habitudes des rédactions à deux niveaux : l'organisation interne et les contenus diffusés. S'agissant de l'organisation, le travail se fait d'une part sur base d'une certaine improvisation, les journalistes étant animés d'un sentiment très fort qu'il faut informer, même s'ils ne 
comprennent pas encore vraiment ce qu'il se passe; d'autre part en se reposant sur leurs pratiques habituelles et sur une certaine expérience, même si les professionnels peuvent manquer de références de comparaison (Konow Lund et al., 2016). La pratique professionnelle réflective (Koljonen et al., 2011), grâce à laquelle les journalistes s'interrogent sur leurs actions et apprennent à partir de leur expérience, a permis à la plupart des rédactions web en Belgique francophone que nous avons interrogées d'améliorer leur dispositif organisationnel en situation de crise liée au terrorisme. Enfin, compte tenu des limites liées à la méthode de l'entretien, et bien que difficile à mettre en place en raison du caractère imprévu de ce type d'événement, il nous semble qu'observer directement et en temps réel les contenus produits sur le Web en période de crise constituerait une piste intéressante pour approfondir notre analyse lors de futures recherches.

\section{BIBLIOGRAPHIE}

Luc Albarello, Apprendre à chercher, l'acteur social et la recherche scientifique, De Boeck \& Larcier, Bruxelles, 1999.

Daniel Benne, Digital Media and Reporting Conflict. Blogging and the BBC's Coverage of War and Terrorism, New York, Routledge, 2013.

Dan Berkowitz, « Non-Routine News and Newswork : Exploring a What-a-Story », Journal of Communication, 42(1), 1992, pp. 82-94.

Nicola Bruno, « Tweet first, verify later? How real-time information is changing the coverage of worldwide crisis events ", in Reuters Institute for the Study of Journalism, 2010-2011.

Béatrice Damian-Gaillard, Franck Rebillard, Nikos Smyrnaios, « La production de l'information web : quelles alternatives? Une comparaison entre médias traditionnels et pure-players de l'internet ». Communication au colloque the New Media and Information, Athènes, 2009.

Amandine Degand, Le journalisme face au web : Reconfiguration des pratiques et des représentations professionnelles dans les rédactions belges francophones, Presses universitaires de Louvain, Louvainla-Neuve, 2012.

Amandine Degand, Benoît Grevisse (dir.), Journalisme en ligne. Pratiques et recherches, De Boeck, Bruxelles, 2012.

Gérard Derèze, Méthodes empiriques de recherche en communication, Editions De Boeck Université, Bruxelles, 2009.

Yannick Estienne, Le journalisme après internet, L'Harmattan, Paris, 2007.

Paul Grabowicz, « Tutorial : the transition to digital journalism », UC Berkley Graduate School of Journalism, Advanced Media Institute, 2014. [en ligne] https:// multimedia.journalism.berkeley.edu/tutorials/digital-transform, consulté le 15 janvier 2017.

Muhammad Zubair Iqbal, «The media-terrorism symbiosis : a case study of Mumbai attacks ", in Asian Journal of Communication, vol. 25, 2015. 
Maria Konow Lund, Eva-Karin Olsson, « When Routines are Not Enough. Journalists' crisis management during the 22/7 domestic terror attack in Norway », in Journalism Practice, vol. 6, 2016, p. 358-372.

Kari Koljonen, Jari Väliverronen, « Crisis journalism at a crossroads ? Finnish journalist's reflections on their profession after two school shooting cases ", in Journalism Practice, vol. 5, 2011, p. 719-734.

Eva-Karin Olsson, « Rule regimes in news organization decision making. Explaining diversity in the actions of news organizations during extraordinary events », in Journalism, 10(6), 2009, pp. 758-776.

Eva-Karin Olsson, « Defining Crisis News Events », in NORDICOM Review, 31(1), 2010, pp. 87-101.

Zizi Papacharissi, Maria Oliveira, « News frames terrorism : A comparative analysis of frames employed in terrorism coverage in U.S. and U.K. Newspapers », in International Journal of Press/ Politics, vol. 13, 2008, p. 52-74.

Nathalie Pignard-Cheynel, Arnaud Mercier, « Mutations du journalisme à l'ère du numérique : un état des travaux », in Revue française des sciences de l'information et de la communication volume 5, mis en ligne le 17 juillet 2014. [En ligne], https://rfsic.revues.org/1097, consulté le 20 juin 2016.

Raymond Quivy, Luc Van Campenhoudt, Manuel de recherche en sciences sociales, 2e édition, Dunod, Paris, 1995.

\section{NOTES}

1. L'analyse de l'audience a été réalisée à partir de données certifiées par le Centre d'Information sur les Médias (CIM). Celles-ci couvrent tous les sites d'informations belges francophones étudiés, à l'exception du site de la télévision bruxelloise Bx1. Pour ce média, nous disposons d'informations fournies par la rédactrice en chef du site internet. Les données du CIM concernant Sudpresse agrègent les chiffres des différents sites des journaux régionaux du groupe : Sudinfo.be, Lacapitale.be, Nordeclair.be, Lameuse.be, Laprovince.be, Lanouvellegazette. be. Les données du Vif.be sont agrégées à celles du site du Knack, newsmagazine flamand appartenant au même propriétaire, l'entreprise Roularta.

2. Les médias considérés comme «de référence» sont le service audiovisuel privé RTL, les journaux quotidiens nationaux Le Soir et La Libre, et la RTBF, service audiovisuel public. Lors des attentats et du Lockdown, Lesoir. be, RTBF.be, RTL.be, et Sudpresse sont les sites les plus visités, suivis de la DH.be, Lalibre. be et 7sur7. Viennent ensuite Knack-Levif, site lié aux news magazines du même nom, et Lavenir.net, adossé au quotidien régional L'Avenir. Les sites de L'Écho et de Newsmonkey bénéficient d'audiences largement inférieures, et connaissent les hausses les moins marquées.

3. La rédactrice en chef de la télévision bruxelloise $B \times 1$, pour laquelle nous ne disposons pas de données certifiées par le CIM, affirme, elle aussi, que le site Bx1.be a connu un trafic intense au cours de ces deux périodes, jusqu'à multiplier son audience habituelle par six pendant le Lockdown.

4. Le 22 mars, les visites provenant de l'étranger représentent $43 \%$ de l'audience du site Lesoir.be, $40 \%$ de celle de Lalibre.be, et $38 \%$ du site RTBF.be. La répartition du nombre total de visites «hors Belgique» montre que ce lectorat se dirige en priorité vers les médias «nationaux».

5. Bien qu'imparfait, car il ne reflète pas les évolutions du journalisme liées à l'influence des nouvelles technologies de l'information et de la communication (TIC), dont le web, l'adjectif 
traditionnel permet de désigner à la fois les médias de presse écrite, radio, et télévision. (Degand, 2012).

6. Le site DH.be du quotidien La Dernière Heure, qui possède également des éditions régionales, n'est pas intégré spécifiquement à notre étude : La Dernière Heure et DH.be partagent de nombreux contenus avec le quotidien et le site de La Libre Belgique, les deux titres appartenant au groupe IPM. Le rédacteur en chef de la Libre.be a cependant apporté un éclairage sur la DH.be dans certaines de ses réponses.

7. Il existe de nombreuses télévisions locales ou spécialisées en Belgique. Nous avons sélectionné Bx1, anciennement Télé Bruxelles, en raison de son implantation à Bruxelles et donc au cœur du Brussels Lockdown et des attentats du 22 mars 2016.

8. Pure player est utilisé ici pour désigner un média exclusivement numérique né sur internet. (Damian-Gaillard, et al., 2009). Le terme anglais désigne une entreprise uniquement présente sur le web et, par extension, une entreprise née sur le web et qui développe ensuite une activité sur un autre support.

9. Le live est un format web qui permet aux journalistes de couvrir l'information qui est en train de se produire, grâce à la possibilité d'ajouter en permanence des informations en tête de page, sous la forme d'un «minute par minute». Ce format permet, par exemple, l'inté- gration de textes, de photos, de vidéos, de cartes et de messages publiés sur les réseaux sociaux.

10. Dans cette vidéo, tournée par un témoin, on aperçoit les décombres, des foyers d'incendie, on entend des cris de douleur et des bruits d'alarme : l'atmosphère de détresse, de chaos et de panique est clairement perceptible, sans images-choc de corps humains délabrés. La séquence est encore visible, notamment, sur le site de la RTBF.

11. "The standards of good journalism are here to stay throughout the digital millennium as long as news media outlets are willing to jump on the bandwagon of real-time information without forgetting their main mission : filtering fact from fiction, separating signal from noise and offering trusted and relevant content. "

\section{RÉSUMÉS}

Le Brussels Lockdown et les attentats de Bruxelles ont généré une importante couverture de la part des médias traditionnels francophones belges, notamment au travers de leurs sites internet. Ces deux périodes ont été caractérisées par une augmentation forte et une restructuration de l'audience des sites d'information. À partir de deux vagues d'entretiens semi- directifs auprès de neuf responsables de rédactions web, cet article se penche sur le rôle de ce support dans la couverture du Lockdown et des attentats de Bruxelles par les rédactions traditionnelles francophones belges, sur le plan organisationnel et sur celui de la production de contenu. L'analyse met en évidence un paradoxe: les rédactions tentent de gérer la demande d'instantanéité par une politique web first, mais en période de crise, peu de moyens humains supplémentaires peuvent être alloués au Web dans les rédactions «traditionnelles ».

Both the Brussels Lockdown and Brussels terror attacks generated substantial coverage from traditional French-speaking Belgian news organizations, notably on their websites. These two periods were characterized by a sharp rise and significant restructuring in their website audience. Based on two rounds of semi-structured interviews with nine news websites editors, this article looks into the role played by the Web in the Lockdown and attacks coverage by 
traditional French- speaking Belgian newsrooms, both from an organizational perspective and from a content production angle. Our analysis has highlighted a paradox : newsrooms try to deal with the demand for immediacy with a web first policy, but little additional human resources can be allocated to Web in "traditional" newsrooms during a crisis.

El Brussels Lockdown y los atentados de Bruselas generaron una cobertura significativa por parte de los medios tradicionales francófonos belgas, particularmente en sus paginas Web. Los dos periodos fueron caracterizados por el gran aumento y la restructuración de la audiencia de las paginas de actualidad. Basado en dos rondas de entrevistas semi- estructuradas con nueve responsables de redacciones Web, el presente articulo examina el papel de la Web en la cobertura del Lockdown y de los atentados de Bruselas por parte de las redacciones tradicionales francófonas belgas, al nivel organizacional y de la producción de contenido. Nuestro análisis sugiere una paradoja: las redacciones tratan de manejar la demanda de inmediatez con una política Web first pero, en periodo de crisis, es poco el personal adicional que puede ser asignado al Web en redacciones "tradicionales".

\section{INDEX}

Mots-clés : terrorisme, brusselslockdown, journalisme de crise, web

Keywords : terrorism, brusselslockdown, crisis journalism, web

Palabras claves : terrorismo, brusselslockdown, periodismo de crisis, web

\section{AUTEURS}

\section{QUENTIN JARDON}

Observatoire de Recherche sur les Médias et le journalisme (ORM), Université catholique de Louvain, Lou- vain-la-Neuve, Belgique.

\section{SANDRINE PUISSANT BAEYENS}

Observatoire de Recherche sur les Médias et le journalisme (ORM), Université catholique de Louvain, Lou- vain-la-Neuve, Belgique.

\section{LARA VAN DIEVOET}

Observatoire de Recherche sur les Médias et le journalisme (ORM), Université catholique de Louvain, Lou- vain-la-Neuve, Belgique. 